\title{
Rare benign pleomorphic adenoma of the nose: short study and literature review
}

\author{
Małgorzata Wierzchowska ${ }^{1}$, Magdalena Bodnar², Paweł K. Burduk ${ }^{1}$, Wojciech Kaźmierczak ${ }^{1,3}$, Andrzej Marszałek ${ }^{2,4,5}$ \\ ${ }^{1}$ Chair of Otolaryngology and Laryngological Oncology, Collegium Medicum in Bydgoszcz, Nicolaus Copernicus University in Torun, \\ Poland \\ 2Department of Clinical Pathomorphology, Collegium Medicum in Bydgoszcz, Nicolaus Copernicus University in Torun, Poland \\ ${ }^{3}$ Department of Pathophysiology of Hearing and Balance System, Collegium Medicum in Bydgoszcz, Nicolaus Copernicus University \\ in Torun, Poland \\ ${ }^{4}$ Department of Oncologic Pathology, Poznan University of Medical Sciences, Poznan, Poland \\ ${ }^{5}$ Greater Poland Oncology Center, Poznan, Poland
}

Videosurgery Miniinv 2015; 10 (2): 332-336

DOI: $10.5114 /$ wiitm.2014.47370

\begin{abstract}
Pleomorphic adenoma is one of the most common benign tumors of the major salivary glands. It can also occur in the minor salivary glands, which exist in the nasal cavity. Intranasal pleomorphic adenoma usually originates from glands of the nasal septum mucosa. We present the results of endoscopic endonasal surgery of this pathology. The aim of the study was to present the endoscopic technique for nasal septum pleomorphic adenoma surgery. The retrospective examination of 3 patients was performed. There were 2 women and 1 man. Age ranged from 15 to 46 years. All the patients presented with nasal obstruction and occasional epistaxis for at least for 6 months. We performed endoscopic surgery to remove the tumors. In all cases we dissected the septal perichondrium to achieved free margins of the tumor. The microscopic examination revealed an epithelial and myoepithelial component with tubular structures composed of two cell layers, ducts, and a solid area found in a loose myxochondroid area. Additionally, immunohistochemical staining was performed using antibodies against cytokeratin, Ki-67, and vimentin. Patients' postoperative course was uneventful, and no complications were encountered. No recurrence was present during patients' postoperative visits. Nasal benign pleomorphic adenoma is a rare tumor which should be taken into consideration in the nasal cavity during surgery. The correct histological diagnosis can be confirmed by additional histological studies. Endoscopic endonasal surgery is reserved for small tumors.
\end{abstract}

Key words: endoscopic surgery, pleomorphic adenoma, histopathology, nasal septum.

\section{Introduction}

Pleomorphic adenoma is one of the most common benign tumors of the major salivary glands. It can also occur in the minor salivary glands, which exist in the nasal cavity, hypopharynx, and glands of the larynx or trachea $[1,2]$. Nevertheless, intranasal, especially septal localization of pleomorphic adenoma is quite rare [3-5]. Intranasal pleomorphic adenoma usually originates from glands of the nasal septum mucosa, although most of the salivary glands are located in the lateral nasal wall and concha $[2,5-7]$.

The pathogenesis of the tumor is still unknown. There are some suggestions that the septal origin of the pleomorphic adenoma could be secondary to

\section{Address for correspondence}

Paweł K. Burduk MD, PhD, Ass. Prof., Chair of Otolaryngology and Laryngological Oncology, Collegium Medicum in Bydgoszcz, Nicolaus Copernicus University in Torun, 9 Skłodowska-Curie 9 St, 85-094 Bydgoszcz, Poland, phone: +48 5258546 14, e-mail: pburduk@wp.pl 
Table I. Clinical features of the cases

\begin{tabular}{|lcccccc|}
\hline Case & Age [years] & Gender & Complaint & Location & Shape & Size [mm] \\
\hline 1 & 22 & M & $\begin{array}{c}\text { Nasal obstruction, bleeding, } \\
\text { deformation }\end{array}$ & Left & Lobulated & $22 \times 15 \times 20$ \\
\hline 2 & 46 & F & Nasal obstruction & Left & Hemispheric & $10 \times 7 \times 9$ \\
\hline 3 & 15 & F & $\begin{array}{c}\text { Nasal obstruction, bleeding, } \\
\text { deformation, pain }\end{array}$ & Right & Hemispheric & $25 \times 10 \times 22$ \\
\hline
\end{tabular}

F-Female, $M-$ male.

misplaced embryonic ectoderm cells, a remnant of the vomeronasal organ or could be induced by a viral infection [5].

Nasal pleomorphic adenomas differ from major salivary gland pleomorphic adenomas as they contain higher cellularity and epithelial component with a low stromal one. These tumors also lack a capsule $[1,4,7,8]$. Histopathology with immunohistochemical examination is the only method suitable to provide definite diagnosis to differentiate a benign from a malignant lesion $[1,2,6,7,9]$.

\section{Case report}

The retrospective examination of 3 patients diagnosed and treated at the Department of Otolaryngology and Laryngological Oncology Collegium Medicum Nicolaus Copernicus University was performed. There were 2 women and 1 man. Age ranged from 15 to 46 years old (Table I). None of them had exposure to cytotoxic chemical agents in the medical history. All the patients presented with nasal obstruction and occasional epistaxis at least for 6 months (Table I). There was no history of visual defect, weight loss or fever. In 2 cases (patients 1 and 3) we observed nasal deformity, and in 1 case (patient 3 ) there was pain due to rapid enlargement of the mass after the biopsy. All the tumors had one side of the nasal cavity obturated (in 2 cases the left side and in 1 the right side) (Table I). Anterior rhinoscopy examination with ridged endoscopy revealed a $1 \mathrm{~cm}$ to $2.5 \mathrm{~cm}$ pathological mass filling the nasal cavity. The surface of the tumors was covered with respiratory epithelium without ulceration or bleeding. Computed tomography scans showed an expansive mass arising from the septum in all cases (Photo 1). No evidence of bone destruction was found.

Histopathological examinations were performed by two independent pathologists in the Department of Pathology, Collegium Medicum Nicolaus Copernicus University, on $\mathrm{H}+\mathrm{E}$ sections from formalin- fixed paraffin-embedded tissue samples processed according to routine diagnostic procedures. Additionally, the immunohistochemical staining was performed on $3 \mu \mathrm{m}$ thickness paraffin tissue sections proceeded automatically in Autostainer48Link (Dako, Glostrup, Denmark) using primary mouse monoclonal antibodies against cytokeratin (clone AE1/AE3, Dako), Ki-67 (clone MIB-1, Dako), and vimentin clone V9, Dako). The antibody complex was detected using EnVisionFlex (Dako) and localized according to the presence of a brown reaction product using DAB as a chromogen.

We performed endoscopic surgery to remove the tumors. In all cases we dissected the septal perichondrium to achieve free margins of the tumor. We used cotton gauze with ointment nasal packing for 2 days after surgery.

The microscopic examination revealed the epithelial and myoepithelial component with tubular structures composed of two cell layers, ducts, and a solid area found in a loose myxochondroid area (Photos $2 \mathrm{~A}-\mathrm{C}$ ).

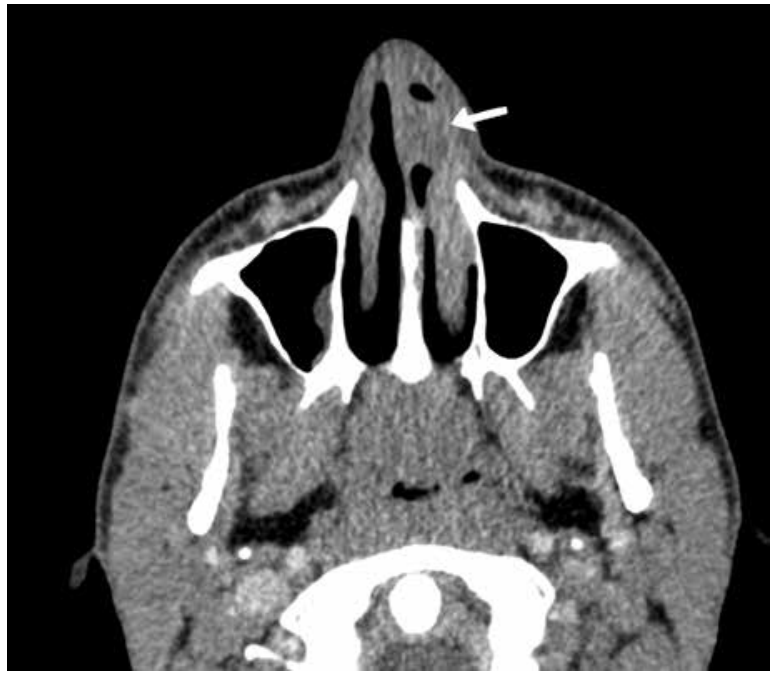

Photo 1. The CT scan showed an expansive mass arising from the nasal septum $(\leftarrow)$ 
The immunohistochemical staining revealed weak expression of cytokeratin AE1/AE3 in the epithelial components. Weak expression of AE1/AE3 cytokeratins was found in basaloid epithelial cells of tubules and cribriform structures, in the mesenchy-
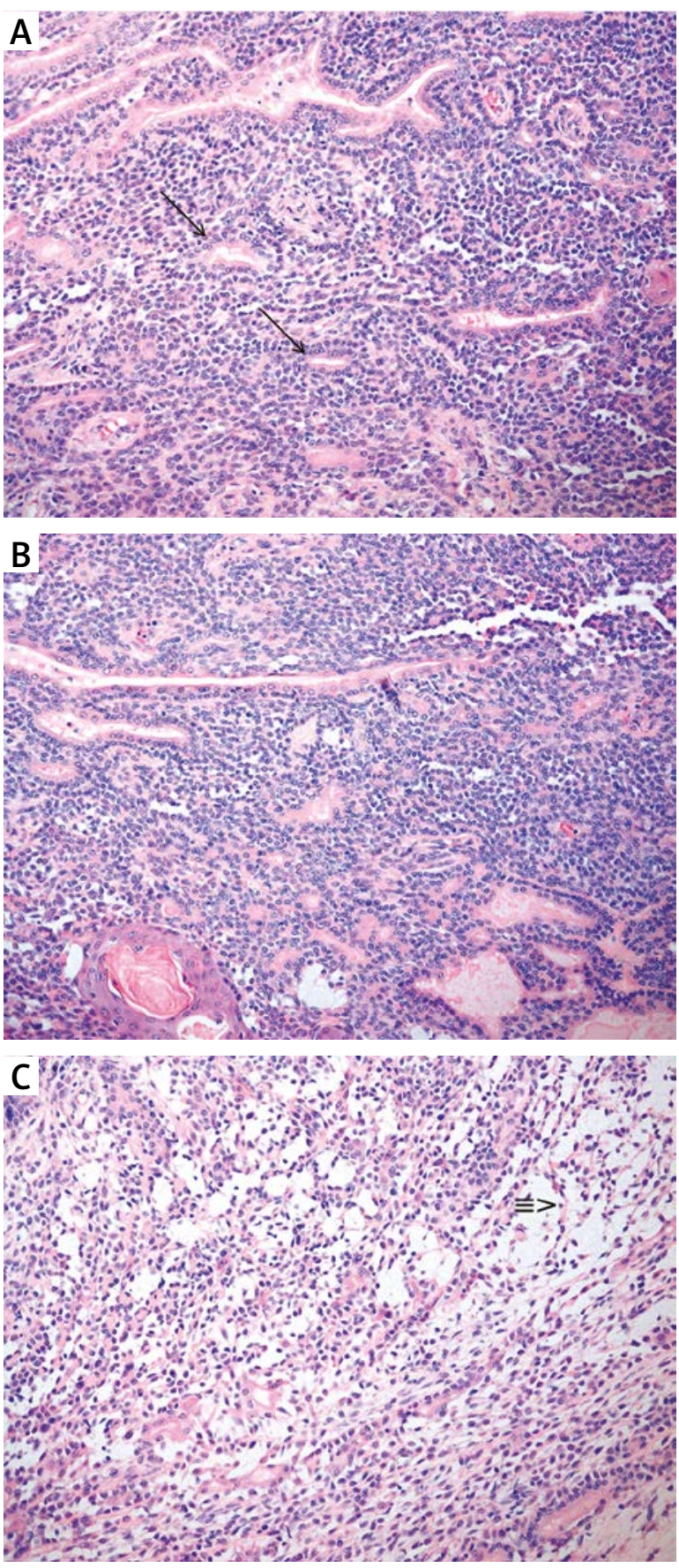

Photo 2 A-C. Microphotographs of epithelial cells forming ducts $(\rightarrow)$, myxoid background $(\equiv)$. $\mathrm{H}+\mathrm{E}$ staining; primary objective magnification $10 \times$ mal cells, as well as in the area of squamous epithelium. Moreover, in the epithelial component with squamous metaplasia, moderate expression of AE1/ AE3 cytokeratins was seen. Strong vimentin expression was confirmed in the myoepithelial cells, as well as in the mesenchymal component.

In the 3 examined cases, a low mitotic index (estimated by Ki-67 expression) was revealed. Expression of Ki-67 was revealed in 1-5\% of epithelial and mesenchymal cells, and in $1 \%$ of the area of squamous metaplasia (Photo 3).

Patients' postoperative course was uneventful, and no complications were encountered. No recurrence was present during patients' visits at 6 months (patient 1), 8 years (patient 2) and 5 years (patient 3 ) after surgery.

\section{Discussion}

The histogenesis of pleomorphic adenoma is generally considered to arise from the epithelial and myoepithelial cells [6]. Pleomorphic adenoma is observed in major salivary glands, but in about $8 \%$ to $10 \%$ of cases it develops in the minor ones $[6,7]$. Mostly, these tumors are benign, and only $1 \%$ are seen in the nasal cavity or epipharynx [6].

The presenting symptoms are usually nasal obstruction and occasionally bleeding. Sometimes we have observed external nasal deformity, nasal swelling or pain $[2,5,8]$. Pleomorphic adenoma of the nasal cavity usually occurred in persons in the third to the sixth decades of life, but sometimes it developed in teenagers, as we observed in one of our cases [7]. The tumor can range in size from $0.5 \mathrm{~cm}$ to $7 \mathrm{~cm}$ $[2,6,8]$. Radiological diagnostics is necessary before surgery, and it could be useful to establish the diagnosis. Computed tomography scans revealed the tumor involvement location (septum or lateral nasal wall) and could suggest an aggressive type if destruction of bone is seen [2, 3, 7-11]. Nuclear magnetic resonance imaging usually showed a heterogeneous mass, isointense to brain on T1-weighted images. The tumor of pleomorphic adenoma showed curvilinear enhancement with unenhanced small foci on fat-suppressed contrast enhanced T1 (cystic or myxoid component of the tumor) $[6,7,10]$.

Compagno and Wong reported their findings from an analysis of clinicopathologic features in 40 cases of intranasal pleomorphic adenoma [4]. They found that histological appearances of this tumor were similar to those of the major salivary 

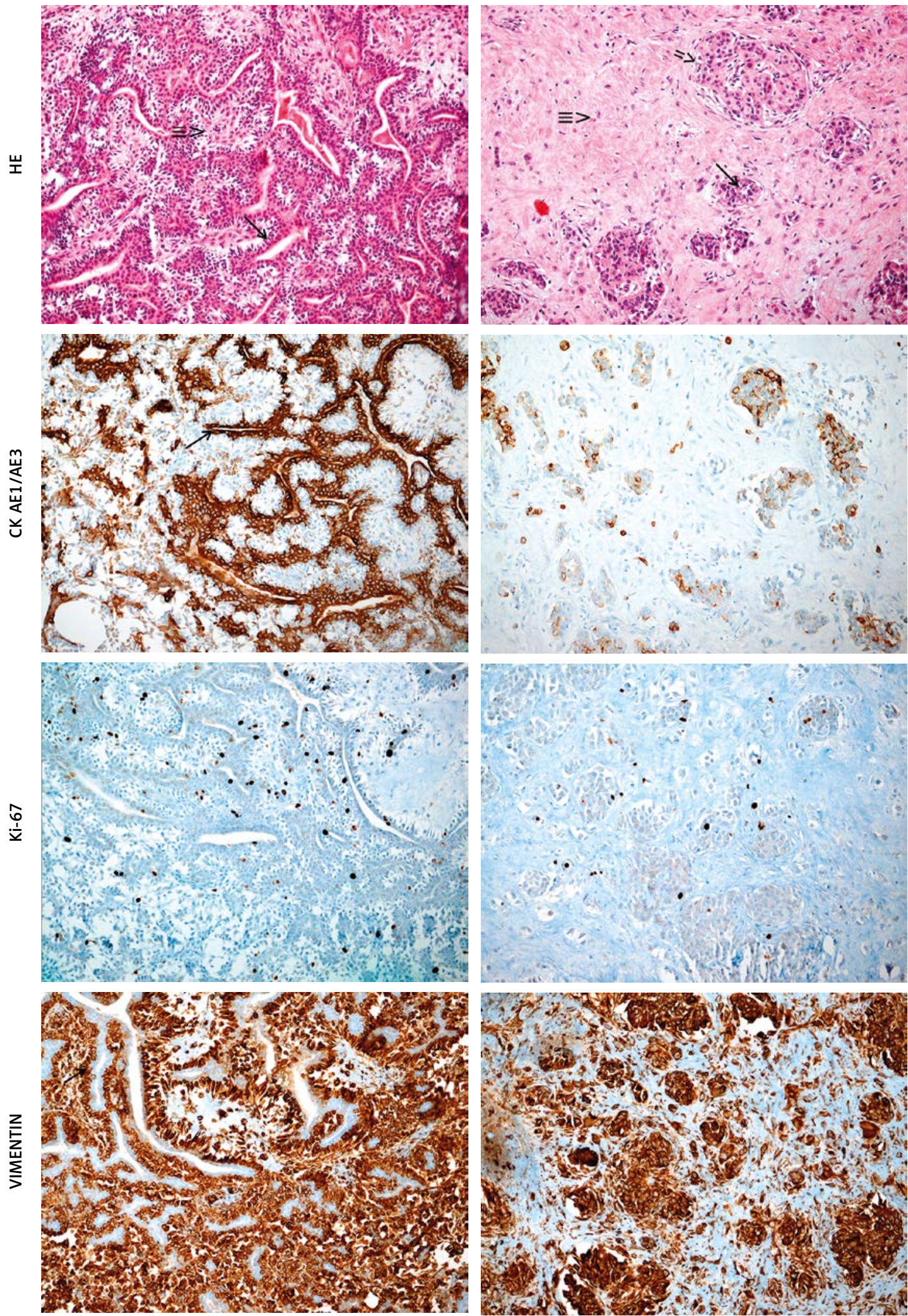

Photo 3. Microphotographs of pleomorphic adenoma in nasal cavity. Ducts $(\rightarrow)$, solid sheets of cells $(=>)$ in myxoid tissue ( $\equiv)$. Immunohistochemical staining of AE1/AE3 cytokeratin, Ki-67 and vimentin $(\rightarrow$ myoepithelial cells). $H+E$ staining; primary objective magnification $10 x$ 
glands. However, intranasal pleomorphic adenomas differed by being of higher cellularity with little or no stromal components [4]. Sometimes these tumors lack a capsule [2]. These histological findings could resemble malignant tumors, such as malignant mixed tumor $[2,12]$. On the other hand, the predominance of epithelial elements rather than stromal may account for the low recurrence rate in this location $[8,13]$. Neoplasms originating from the nasal septum have a higher risk of malignancy. Occasionally, pleomorphic adenoma of the nasal cavity can behave in a malignant fashion like carcinoma ex pleomorphic adenoma. Immunohistochemical staining with antibodies of various cytokeratins, glial fibrillary acidic protein, S100 protein, $\alpha$-smooth muscle actin, vimentin and the Ki67 proliferation marker could confirm the final diagnosis $[1,2,6,7,14]$.

Surgical treatment methods include endonasal endoscopic surgery and lateral rhinotomy. The method of surgical approach depends on the size of the tumor and its location. If there is a low rate of recurrence and relatively small size up to $2.5 \mathrm{~cm}$ in diameter, as in our material, endoscopic resection is appropriate $[2,7,11]$.

\section{Conclusions}

Nasal benign pleomorphic adenoma is a rare tumor which should be taken into consideration in the nasal cavity during surgery. The correct histological diagnosis can be confirmed by additional histological studies. Endoscopic endonasal surgery is reserved for small tumors.

\section{Acknowledgments}

The authors would like to thank the patients for taking part in this study.

\section{Conflict of interest}

The authors declare no conflict of interest.

\section{References}

1. Hirai S, Matsumoto T, Suda K. Pleomorphic adenoma in nasal cavity: immunohistochemical study of three cases. Auris Nasus Larynx 2002; 29: 291-5.

2. Baglam T, Durucu C, Cevik C, et al. Giant pleomorphica adenoma of the nasal septum. Ind J Otolaryngol Head Neck Surg 2011; 63: 393-5.

3. Golz A, Ben-Arie Y, Fradis M. Pleomorphic nasoseptal adenoma. J Otolaryngol 1997; 26: 399-401.
4. Compagno J, Wong RT. Intranasal mixed tumors (pleomorphic adenomas). A clinicopathologic study of 40 cases. Am J Clin Pathol 1997; 68: 213-8.

5. Narozny W, Przewozny T, Stankiewicz C, et al. Pleomorphic adenomas of the nasal cavity. Otolaryngol Pol 2003; 57: 661-5.

6. Kumagai M, Endo S, Koizumi F, et al. A case of pleomorphic adenoma of the nasal septum. Auris Nasus Larynx 2004; 31 : 439-42.

7. Malinvaud D, Couloigner V, Badoual C, et al. Pleomorphic adenoma of the nasal septum and its relationship with Epstein-Barr virus. Auris Nasus Larynx 2006; 33: 417-21.

8. Tahlan A, Nanda A, Nagarkar N, et al. Pleomorphic adenoma of the nasal septum: a case report. Am J Otolaryngol 2004; 25 : 118-20.

9. Yazibene Y, Ait-Mesbah N, Kalafate S, et al. Degenerative pleomorphic adenoma of the nasal cavity. Eur Ann Otorhinolaryngol Dis 2011; 128: 37-40.

10. Oztürk E, Sağlam O, Sönmez G, et al. CT and MRI of an unusual intranasal mass: pleomorphic adenoma. Diagn Interv Radiol 2008; 14: 186-8.

11. Gotlib T, Osuch-Wójcikiewicz E, Held-Ziółkowska M, et al. Endoscopic transnasal management of sinonasal malignancies our initial experience. Videosurgery Miniinv 2014; 9: 131-7.

12. Batsakis JG. Tumors of the head and neck. 2nd ed. Wiliams and Wilkins, Baltimore 1984; 76-99.

13. Manning JT, Batsakis JG. Salivary-type neoplasm of the sinonasal tract. Ann Otol Rhinol Laryngol 1991; 100: 691-4.

14. Nonomura N, Niijima H, Kimura O, et al. Immunohistochemical study of pleomorphic adenoma of the nasal septum. Auris Nasus Larynx 1992; 19: 125-31.

Received: 20.09.2014, accepted: 12.11.2014. 